\title{
Intrinsic and extrinsic corrugation of monolayer graphene deposited on $\mathrm{SiO}_{2}$
}

\author{
V. Geringer ${ }^{1,3}$, M. Liebmann ${ }^{1,3}$, T. Echtermeyer ${ }^{2}$, S. Runte ${ }^{1,3}$, \\ M. Schmidt ${ }^{1,3}$, R. Rückamp ${ }^{1,3}$, M. C. Lemme ${ }^{2}$, and M. Morgenstern ${ }^{1,3}$ \\ ${ }^{1}$ II. Institute of Physics, RWTH Aachen University, \\ Otto-Blumenthal-Straße, 52074 Aachen \\ ${ }^{2}$ Advanced Microelectronic Center Aachen (AMICA), AMO GmbH, \\ Otto-Blumenthal-Straße 25, 52074 Aachen, \\ 3 JARA: Fundamentals of Future Information Technology
}

(Dated: November 5, 2018)

\begin{abstract}
Using scanning tunneling microscopy (STM) in ultra high vacuum and atomic force microscopy, we investigate the corrugation of graphene flakes deposited by exfoliation on a $\mathrm{Si} / \mathrm{SiO}_{2}(300 \mathrm{~nm})$ surface. While the corrugation on $\mathrm{SiO}_{2}$ is long-range with a correlation length of about $25 \mathrm{~nm}$, some of the graphene monolayers exhibit an additional corrugation with a preferential wave length of about $15 \mathrm{~nm}$. A detailed analysis shows that the long range corrugation of the substrate is also visible on graphene, but with a reduced amplitude, leading to the conclusion that the graphene is partly freely suspended between hills of the substrate. Thus, the intrinsic rippling observed previously on artificially suspended graphene can exist as well, if graphene is deposited on $\mathrm{SiO}_{2}$.

PACS numbers: 68.55.J-, 68.37.Ef, 68.37.Ps, 68.65.-k
\end{abstract}

Since it was believed, based on the Mermin-Wagner theorem, that two-dimensional (2D) crystals are not stable at finite temperature [1, it came as a surprise that monolayer graphene could be stabilized on a $\mathrm{Si} / \mathrm{SiO}_{2}$ substrate [2, 3, 4. Due to its peculiar properties like, e.g., a linear dispersion leading to Klein tunneling [5, high room-temperature mobility allowing quantum Hall steps at $300 \mathrm{~K}$ [6], a low spin-orbit interaction beneficial for spintronic devices [7] or tunable spin-polarized edge states [8, graphene studies have become a major issue in solid-state physics. Already the first transport results not exhibiting weak localization lead to the speculation of a curved surface [9] acting as a phasebreaking field [10. Such a curvature was indeed observed by microscopic electron diffraction of suspended monolayer graphene sheets [11. The lateral wavelength of the isotropic curvature is estimated to be $\lambda=10-25 \mathrm{~nm}$ with an amplitude of $A \simeq 1 \mathrm{~nm}$. The rippling has been reproduced theoretically by Monte-Carlo simulations with a preferential wavelength of $8 \mathrm{~nm}$ barely depending on temperature [12. It is argued that the anharmonic coupling between bending and stretching modes in graphene causes the rippling and is responsible for the stability of the 2D crystal. Moreover, it has been shown experimentally that rippling can ultimately limit the mobility of graphene at $300 \mathrm{~K}$, if defects are avoided [13.

However, the corrugation properties of graphene deposited on a substrate as typically used in transport experiments are not clarified. In particular, previous scanning probe studies [14, 15, 16, 17] revealed only corrugations, which are attributed to the roughness of the underlying substrate, except for a torn flake manipulated by AFM [16. Thus, it is unclear, if the intrinsic tendency for rippling persists on the substrate, thereby ultimately limiting mobility and influencing weak localization, which has meanwhile been found, at least, for some samples [16. Here, we demonstrate that a regular short wave- length corrugation with $\lambda \simeq 15 \mathrm{~nm}$ and $A \simeq 1 \mathrm{~nm}$, which is not induced by the substrate, can even prevail the substrate corrugation. Since $\lambda$ and $A$ are close to the values found on suspended graphene [11, we attribute this additional corrugation to the intrinsic rippling of graphene [12. Moreover, we find that the long-range corrugation implied by the substrate has lower amplitude on graphene than on $\mathrm{SiO}_{2}$ suggesting that our high-mobility graphene is partly suspended between hills of the substrate, which might favor the development of the intrinsic short-scale rippling.

The graphene sample is fabricated by the mechanical exfoliation technique as described in 2, 18. Using an optical microscope, a graphene flake containing a monolayer region is identified. Raman spectroscopy is used to confirm the number of layers with the help of the $2 \mathrm{D}$ line [19]. Fig. 1(a) shows three spectra measured in different areas of the flake visible in Fig. 1(b). In addition to a large monolayer area, we find smaller bi- and multilayer areas as marked by $1 \mathrm{~L}, 2 \mathrm{~L}$ and $\mathrm{MuL}$, respectively. Next, gold contacts with a $10 \mathrm{~nm}$ Cr seed layer were deposited and structured with a lift-off process. Except for one side, the graphene sample is completely surrounded by the gold electrode as shown in Fig. 1(b). The mobility of graphene samples prepared identically but with several contacts is $\mu=1-1.5 \mathrm{~m}^{2} /(\mathrm{Vs})$ at $300 \mathrm{~K} 20$, i.e. comparable with high-mobility values obtained by other groups [13, 21. In order to remove residual resist and adsorbates, the sample was rinsed in isopropanol and acetone, baked out to $150^{\circ} \mathrm{C}$ in air for four hours and, additionally, baked out to the same temperature in ultrahigh-vacuum (UHV) for three hours. The gold film served as the electrical contact for scanning tunneling microscopy (STM) and was used to prepare the STM tip (etched W) by voltage pulses. In order to find the graphene within the UHV-STM, we used an optical microscope with a focal length of $30 \mathrm{~cm}$ and $5 \mu \mathrm{m}$ lateral 


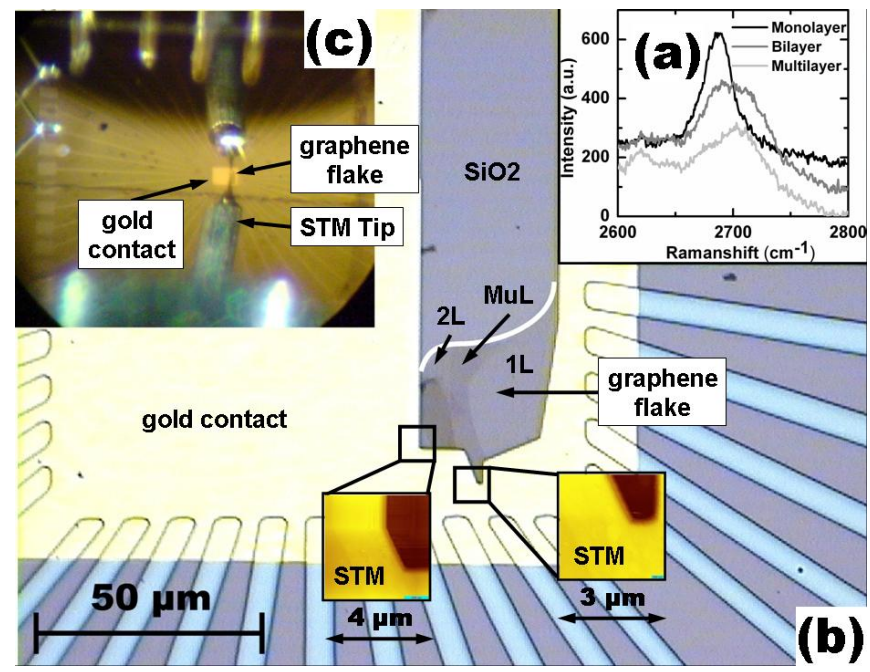

FIG. 1: (Color online) (a) Raman spectra using laser light of wavelength $532.1 \mathrm{~nm}$. The spectra are recorded on different areas of the graphene flake and the attribution to monolayer, bilayer and multilayer is marked. (b) Optical image of the graphene sample with gold contacts. The different areas of monolayer $(1 \mathrm{~L})$, bilayer $(2 \mathrm{~L})$ and multilayer $(\mathrm{MuL})$ as identified by Raman spectroscopy are indicated. Insets show two STM images acquired at the edge of the gold contact, which are used for orientation. (c) Microscopic image of the STM tip in tunneling contact with the graphene in UHV recorded with a long-range optical microscope.

resolution. Fig. 11(c) shows a microscopic image of the tip approaching the graphene flake. The home-built STM features an $x y$ stage for lateral positioning and operates in $\operatorname{UHV}\left(2 \cdot 10^{-7} \mathrm{~Pa}\right)$ as described elsewhere $22,23$. The $x y$ stage has been checked to move accurately within 10 $\%$. Topographic images are recorded applying a bias $V$ to the tip. Spectroscopic $d I / d V$ curves are measured by lock-in technique using a modulation voltage $V_{\text {mod }}$ after stabilizing the tip at voltage $V_{\text {stab }}$ and current $I_{\text {stab }}$. STM images obtained at the intentionally irregular edge of the gold contact are displayed in the insets of Fig.1 (b). They are used for orientation by STM and aid in finding monolayer, bilayer and multilayer areas on the flake.

Atomic force microscopy images are taken under ambient conditions in tapping mode using either a commercial cantilever with a silicon tip or an ultrasharp tungsten tip with a tip radius of $1 \mathrm{~nm}$, which is attached at the bottom of a Si cantilever 24]. Imaging has been carried out in the attractive regime [25] with an oscillation frequency slightly above resonance.

Fig. 2(b) and (c) show two atomically resolved STM images recorded on the monolayer $(1 \mathrm{~L})$ and the multilayer $(\mathrm{MuL})$ of graphene, respectively. One observes a hexagonal pattern on the monolayer and a triangular pattern on the multilayer in accordance with previous results [14, 17. The atomic resolution is still perceptible at lower resolution in Fig.2(a) and (d), but an additional irregular corrugation appears. Line sections shown in Fig. 2(e) reveal that the corrugation height on this monolayer region is about $0.6 \mathrm{~nm}$, a factor of three larger than on the multilayer region. Notice that corrugation heights pretended by charged defects are typically smaller by more than an order of magnitude [26]. A representative $d I / d V /(I / V)$ curve of the monolayer, which is known to represent the local density of states (LDOS) [27, is shown in Fig. 2(f). As expected for graphene, it shows a V-like shape with the Dirac point at about $V=-20 \mathrm{mV}$. It does not change significantly across the area depicted in Fig. 2(d). Note that graphene on $\mathrm{SiC}(0001)$ exhibits a more complicated $d I / d V$ spectrum [28, 29, 30]. Note also, that we did not observe the phonon gap found at $4 \mathrm{~K}$ recently [17, although we used several different micro- and macrotips. This is tentatively attributed to the different temperature of the two experiments. The good representation of the graphene LDOS by $d I / d V /(I / V)$ and the continuous atomic resolution across Fig. 2(d) demonstrates the absence of resist on this part of the surface. Indeed, we found several large areas without resist, but still also areas where a remaining resist is apparent within the STM images. The fact that the Dirac point is observed close to $0 \mathrm{~V}$ shows a negligible influence of charging adsorbates, which are probably removed in UHV.

Fig. 3(a) shows a large area STM image of the graphene monolayer. One observes a rather regular corrugation with amplitudes of more than $1 \mathrm{~nm}$ and a preferential distance between hills of about $15 \mathrm{~nm}$. The histogram 32 is Gaussian with a full width at half maximum (FWHM) of $0.78 \mathrm{~nm}$. The rms roughness is determined to be $0.36 \mathrm{~nm}$. The latter two values fluctuate across the monolayer area by about $25 \%$ (average rms roughness $0.32 \mathrm{~nm}$ ), but the corrugation length scale remains constant. For comparison, Fig. 3(c) shows an AFM image of the bare $\mathrm{SiO}_{2}$ surface recorded in tapping-mode. It is obvious that the corrugation on the substrate is lower and exhibits a larger length scale than the corrugation on graphene. The FWHM of the histogram of Fig. 33(c) is $0.48 \mathrm{~nm}$ and the rms roughness is $0.22 \mathrm{~nm}$ (average of all data $(0.25 \pm 0.05) \mathrm{nm})$. We used four different tips, including two ultrasharp tips with a tip radius of $1 \mathrm{~nm}$ [24], but always observed the same corrugation height and length scale, which, in addition, is in good agreement with previous results [15].

Careful inspection of the graphene monolayer in Fig. 3(a) and (b) shows a modulation on top of the shortrange corrugation exhibiting a similar length scale as the corrugation on the $\mathrm{SiO}_{2}$ substrate. In order to disentangle the two contributions, we used two-dimensional autocorrelation functions of the images (a) and (c) 31, which are shown in Fig. 4(a) and (b). Note that both figures use the same color scale, i. e. the intensities are directly comparable. A long-range structure represented by a central spot surrounded by four bright areas is visible in both images although with weaker intensity for graphene (a). Fig. 4 (c) shows the autocorrelation function of the graphene after removing the long-range part by high-pass filtering using a smooth (first order Butterworth) cutoff at wavelength $\lambda=20 \mathrm{~nm}$. A preferential 

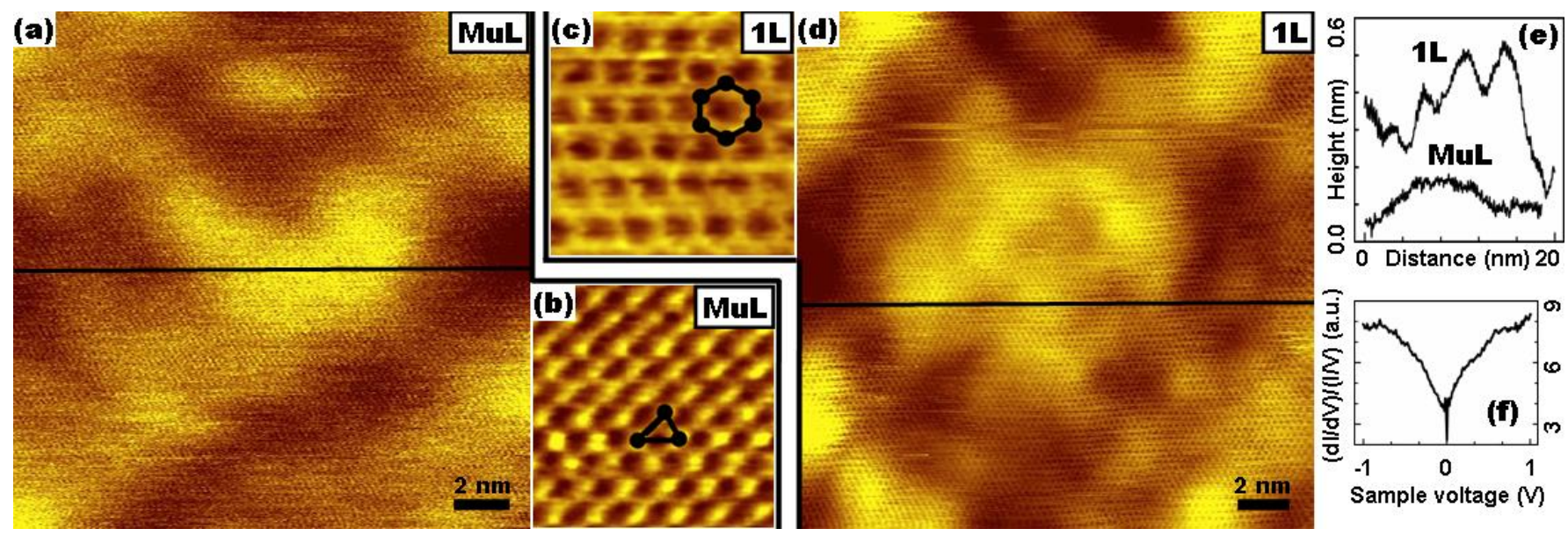

FIG. 2: (Color online) (a) Constant current STM image of multilayer graphene (0.4 V, 258 pA) (raw data). (b) Higher resolution STM image of the same area as in (a) $(0.4 \mathrm{~V}, 198 \mathrm{pA})$. (c) High resolution STM image of graphene monolayer area $(1 \mathrm{~V}, 394 \mathrm{pA})$. (d) Lower resolution STM image of monolayer graphene (0.5 V, $292 \mathrm{pA})$ (raw data). (e) Line section along the lines depicted in $(\mathrm{a})(\mathrm{MuL})$ and $(\mathrm{d})(1 \mathrm{~L})$. (f) Normalized $\mathrm{dI} / \mathrm{dV}$ spectrum of monolayer graphene: $V_{\mathrm{stab}}=0.7 \mathrm{~V}, I_{\mathrm{stab}}=300 \mathrm{pA}$, $V_{\bmod }=20 \mathrm{mV}$.
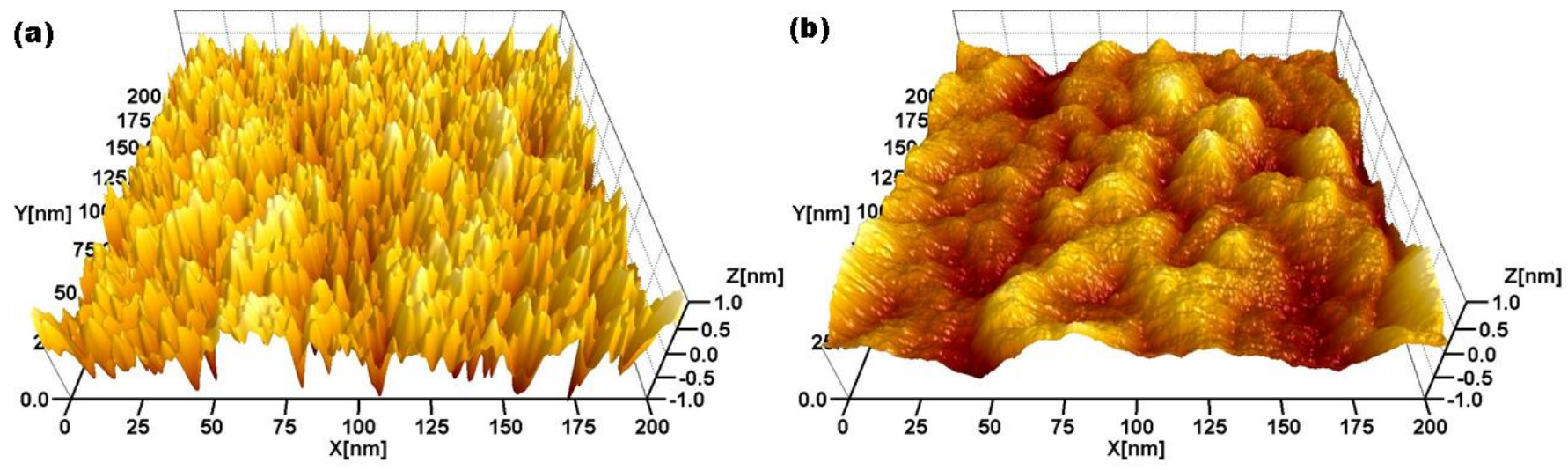

FIG. 3: (Color online) (a), (b) 3D and 2D constant current STM image of monolayer graphene (1 V, 207 pA). (c) 3D tappingmode AFM image of the $\mathrm{SiO}_{2}$ substrate (resonance frequency $326.4 \mathrm{kHz}$, force constant $47 \mathrm{~N} / \mathrm{m}$, excitation frequency $326.5 \mathrm{kHz}$, oscillation amplitude $18 \mathrm{~nm}$, constant amplitude feedback, setpoint 90\%).

short-range distance is clearly visible by the strong spots around the center, but there is additional multiple correlation albeit not with high symmetry. Notice that creep and drift effects are carefully removed from the STMimage using the atomic resolution. We checked the preferential directions visible in both correlation patterns. They are rotating arbitrarily across the $\mathrm{SiO}_{2}$, but we find indication for a preferential orientation on graphene 32. Fig. 4(d) shows radial line sections averaged over all angles of the correlation functions. The $\mathrm{SiO}_{2}$ (black curve) exhibits a correlation length of about $25 \mathrm{~nm}$ comparable to [15] and, in addition, a very weak maximum at about $50 \mathrm{~nm}$ indicating a slight preferential distance between hills. The gray curve shows the radial line section of the high-pass filtered image of graphene visible in Fig. 4(c). It exhibits a damped oscillation with a wave length of about $14 \mathrm{~nm}$ looking very similar to correlation functions of liquids. For comparison, the radial line section of the autocorrelation function of the AFM image after applying the same high-pass filter is shown as a dotted line and does not exhibit any structure. We observe the damped oscillation also after high-pass filtering the original STM image in Fig. 3. (a) and determining the autocorrelation function afterwards. In addition, we checked that the obtained wave length is quite robust with respect to slight parameter changes of the filtering process. Depending on the filtering procedure and the selection of the minima or maxima used for determining the wave length, $\lambda$ is varying by at maximum $\pm 2 \mathrm{~nm}$ with a mean value of $\lambda=15 \mathrm{~nm}$.

From these results, we conclude that monolayer graphene can exhibit a rather regular, liquid-like shortscale corrugation not induced by the substrate. It is similar in height and wavelength to the one observed on suspended graphene 11 and calculated for freely suspended graphene by atomic Monte-Carlo methods [12]. 


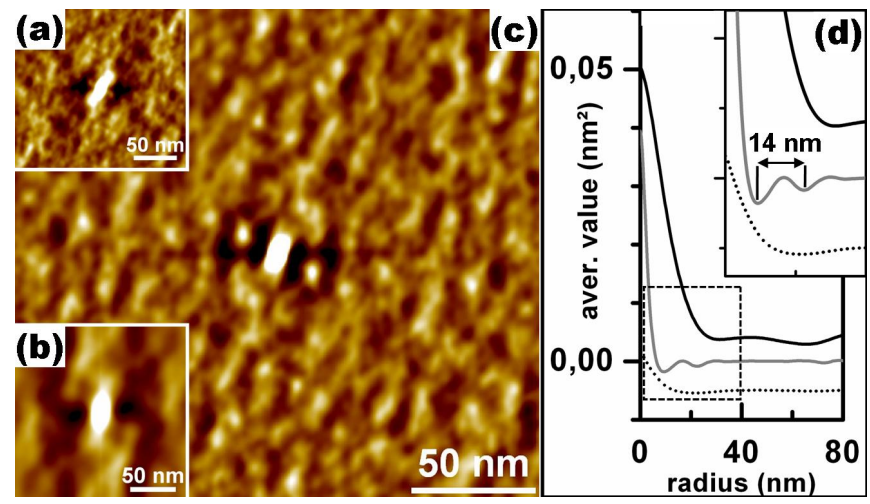

FIG. 4: (Color online) (a) 2D autocorrelation function of the drift corrected STM image of monolayer graphene as shown in Fig. 3(a). (b) 2D autocorrelation function of the AFM image of the $\mathrm{SiO}_{2}$ surface as shown in Fig. 3.(c), same color scale as in (a). (c) FFT high pass filtered image (1. order Butterworth, $20 \mathrm{~nm}$ ) of the 2D autocorrelation function shown in (a). (d) Radial line sections of the unfiltered AFM autocorrelation image (b) (black), of the high pass filtered STM autocorrelation image (c) (gray), and of the high pass filtered AFM autocorrelation image (dotted). The inset shows a larger view of the area marked by the dashed line. The black and gray graphs are offset vertically for clarity.

This observation suggests that the graphene might be partly suspended even on the $\mathrm{SiO}_{2}$. In order to verify this, we compared the Fourier components of the images of graphene and $\mathrm{SiO}_{2}$ within the large wavelengths region $\lambda=40-60 \mathrm{~nm}$ [32. We indeed find that graphene exhibits an up to $40 \%$ lower amplitude at all wavelengths within this wavelength range. This implies that the graphene does not follow the substrate corrugations exactly, but instead includes areas not in contact with the substrate. We believe that this partly free-standing configuration is the prerequisite for the intrinsic rippling of graphene. Indeed, we have also found other graphene flakes prepared nominally identically by the same person which did not show the intrinsic rippling. This highlights that tiny details of the preparation process can have large impacts on the physical properties, maybe explaining the contradicting weak localization properties in different studies [9, 16. A comparison of Raman data of several samples indicates that samples with intrinsic rippling are more frequent than samples without [32]. Future systematic studies using our method to determine the intrinsic rippling are required.

In summary, we have investigated high-mobility graphene flakes on $\mathrm{SiO}_{2}$ by UHV-STM and scanning tunneling spectroscopy. A mesoscopic corrugation decreasing in amplitude with increasing thickness not induced by the substrate is identified. It is rather regular exhibiting liquid-like correlation properties with a preferential wavelength of about $15 \mathrm{~nm}$ and a rms roughness of $0.32 \mathrm{~nm}$. Since the long-range corrugation of the substrate is additionally visible on graphene, but with smaller amplitude than on the substrate, we conclude that the rippled

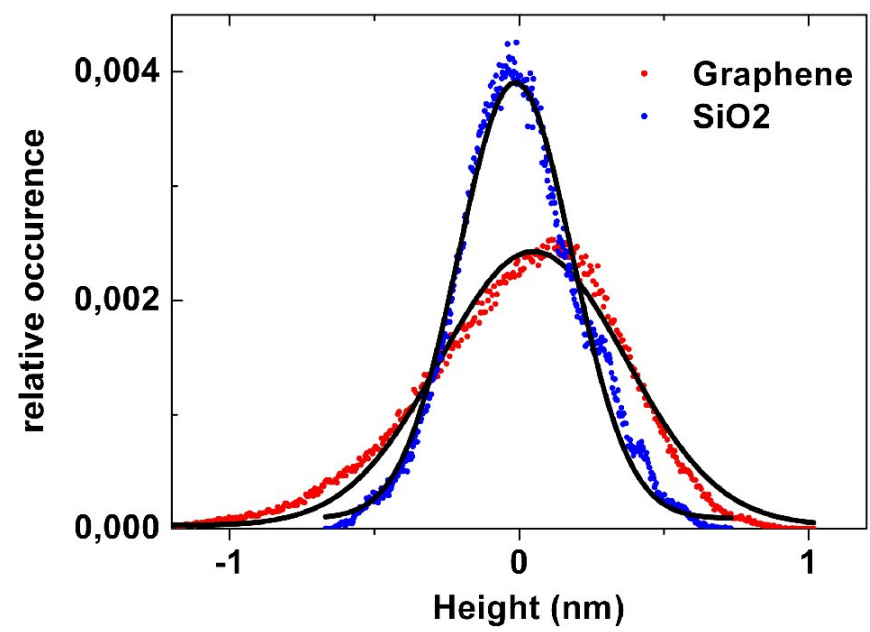

FIG. 5: (Color online) Height histograms of $\mathrm{SiO}_{2}$ (blue) and graphene (red) corresponding to the images in Fig. 3 of the main text. On average, the graphene histogram exhibits a larger FWHM value compared to $\mathrm{SiO}_{2}$.

graphene is partly freely suspended.

We gratefully acknowledge useful discussions with G. Güntherodt, U. D. Schwarz and H. Kurz and financial support by FOR 912, project TP 6 of the Deutsche Forschungsgemeinschaft and by the German Federal Ministry of Education and Research (BMBF) under contract number NKNF 03X5508 ("ALEGRA").

\section{Supplementary information}

\section{Histogram of Corrugation}

Figure 5 shows representative histograms of the height values on the $\mathrm{SiO}_{2}$ substrate and the graphene sample as obtained by atomic force microscopy (AFM) and scanning tunneling microscopy (STM), respectively. The histograms are based on images of $200 \times 200 \mathrm{~nm}^{2}$ including $512 \times 512$ measurement points each. These images are shown in Fig. 3 of the main text. The histogram curves are normalized in order to enclose the same integral area. Gaussian fits are added as guides to the eye. Obviously, the height histogram of Graphene is broader exhibiting a full width at half maximum (FWHM) of $0.78 \mathrm{~nm}$, while $\mathrm{SiO}_{2}$ exhibits a FWHM of only $0.48 \mathrm{~nm}$. While the latter value is quite robust across the surface, the former value is changing by about $25 \%$ across the surface, but is on average larger than the FWHM on $\mathrm{SiO}_{2}$. This demonstrates that the rippling of graphene is not limited to substrate corrugations.

\section{Tip Dependence of Substrate Corrugation}

Figure 6 shows two AFM images of the $\mathrm{SiO}_{2}$ substrate obtained in tapping mode by two different tips. Rep- 
resentative scanning electron microscopy (SEM) images of the tips provided by the supplier are added as insets. They are cross-checked by SEM images after the measurements. These images are in agreement with the supplier images, but due to a moderate background pressure, the ultrasharp W-tip fastly burnt away during SEM imaging. The left AFM image is measured by a cantilever with a Si tip having an apex radius of about $10 \mathrm{~nm}$, while the right AFM image is measured with a $\mathrm{W}$ tip of nominal tip apex radius of $1 \mathrm{~nm}$ mounted at the bottom of the Si tip. Both images exhibit a very similar lateral scale of the corrugation. The rms values are $220 \mathrm{pm}$ and 270 $\mathrm{pm}$, respectively, showing that the tip properties do not significantly change the observed corrugation of the substrate. We cross-checked this result using more than ten images obtained with both tips, which lead to fluctuations in rms values of less than $\pm 10 \%$.

\section{Comparison of Correlation Functions}

Figure 7 shows a comparison of the correlation functions of the topographic images of graphene (top row) and $\mathrm{SiO}_{2}$ (bottom row). The left column shows the correlation functions directly obtained from the topography. The middle and right column show the high-frequency and low-frequency part of the correlation function separated by a smooth first-order butterworth filter with cutoff wavelength $\lambda=20 \mathrm{~nm}$. While the large wavelengths visible in the right columns are quite similar in both images, the rather regular short wavelength pattern (middle column) is only observed on graphene. Note that correlation functions within the same columns are scaled to the same contrast, i. e., their brightness is directly comparable.

\section{Reproducibility of Correlation Functions}

Figure 8 shows three images (top row) obtained in different areas of the monolayer graphene, separated by several $\mu \mathrm{m}$. They exhibit different rms values of $270 \mathrm{pm}, 220$ $\mathrm{pm}$ and $360 \mathrm{pm}$ (left to right), respectively. The middle row shows the corresponding correlation functions. The bottom row shows the same correlation functions after high-pass filtering using the identical procedure as in Figure 3. Notice that the scales of the images are different. While the long-range corrugation (middle row) is oriented in different directions, the short range corrugations (bottom row) exhibit the same wave length of about $15 \mathrm{~nm}$ in all three areas and show a similar orientation as marked by the white lines. The latter indicates that the the short-scale corrugation is related to the atomic directions in graphene. But since the directions of the rippling are fluctuating slightly across the sample similar to the orientations of molecules in liquid crystals, more data are required in order to substantiate the relation between atomic directions and preferred directions of rippling.

Figure 9 shows topographic images of different areas of the $\mathrm{SiO}_{2}$ substrate (top row) and the corresponding correlation functions (bottom row). They exhibit similar length scales of corrugation but clearly different orientations, which might be due to the polishing procedure of the sample.

\section{Wave Length Dependence of Corrugations}

Figure 10 shows the radially averaged Fourrier transforms (FT) of the topographic images of graphene (black curve) and $\mathrm{SiO}_{2}$ (grey curve). The FT curves are based on images of the same lateral size and resolution. The curves are averaged using 10 images of $200 \times 200 \mathrm{~nm}^{2}$ from different areas of the surfaces. Obviously, the corrugation of $\mathrm{SiO}_{2}$ is larger at large wave length (small wave number) down to $30 \mathrm{~nm}$ by up to $66 \%$, while the corrugation on the graphene is larger at smaller wavelengths (larger wave numbers) by up to a factor of 2.6. The individual FTs of different $\mathrm{SiO}_{2}$ areas are nearly indistinguishable, i.e they are not deviating from the width of the grey curve in Fig. 5. However, the FTs of different graphene areas are shifted vertically due to the fluctuating rms value of corrugation, which leads to crossing points of the two curves varying between wavelengths of $20 \mathrm{~nm}$ and $40 \mathrm{~nm}$. Importantly, all of the graphene curves show one crossing point with the averaged $\mathrm{SiO}_{2}$ curve.

\section{Raman investigations on monolayer graphene}

In order to observe the influence of the graphene film morphology on Raman scattering, we compared Raman spectra (laser wave length $532.1 \mathrm{~nm}$ ), taken in identical experimental conditions, of a monolayer flake which is partly suspended and one that follows the substrate (Fig. 11). The surface structure of these samples was determined using STM measurements (Insets Fig. 117). The Raman spectra were recorded before and after the STM measurements giving the same result. A difference between both spectra regarding the positions of the $2 \mathrm{D}$ and $\mathrm{G}$ peaks is clearly visible. The wave number of the $2 \mathrm{D}$ peak is $2686.7 \mathrm{~cm}^{-1}$ for the partly suspended graphene flake and $2670.5 \mathrm{~cm}^{-1}$ for the flake following the substrate. Hence, it results that the change in morphology causes a shift of the 2D peak by $16.2 \mathrm{~cm}^{-1}$ (Fig. 11 ). A similar effect was observed at the $\mathrm{G}$ peak which is shifted by $5.5 \mathrm{~cm}^{-1}$ to lower values for the flake following the substrate (Fig. 11p). The D peak (about 1350 $\mathrm{cm}^{-1}$ ) is barely visible for both samples indicating a good quality of our graphene. Raman spectra of several of our graphene samples (about 15) prepared in nominally identical conditions show more frequently a non shifted 2D line with an average value of $2692.4 \pm 5.3 \mathrm{~cm}^{-1}$. Hence, we conclude that our samples mostly exhibit intrinsic rippling. If we compare with wave numbers of the $2 \mathrm{D}$ line 


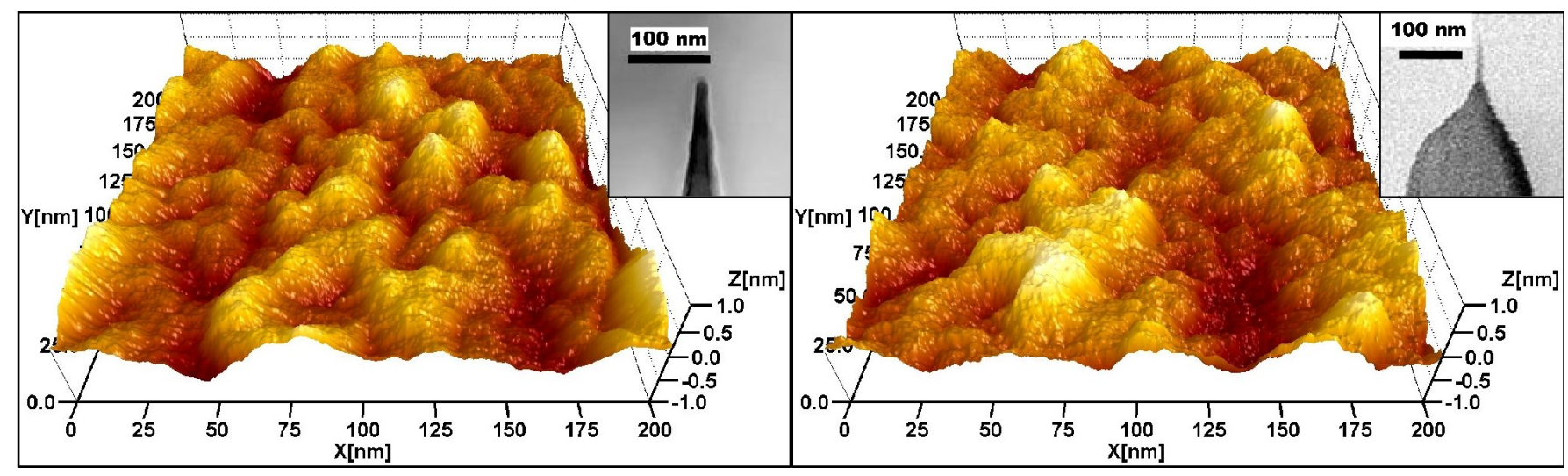

FIG. 6: (Color online) AFM images of $\mathrm{SiO}_{2}$ with tips of different radii: Si tip NSC15 with $10 \mathrm{~nm}$ (a) and W/Si tip DP15/HiResW/AlBS with $1 \mathrm{~nm}$ (b), respectively. Both images show the same color scale. Insets: SEM images of similar tips, with kind permission of MikroMasch (www.spmtips.com).
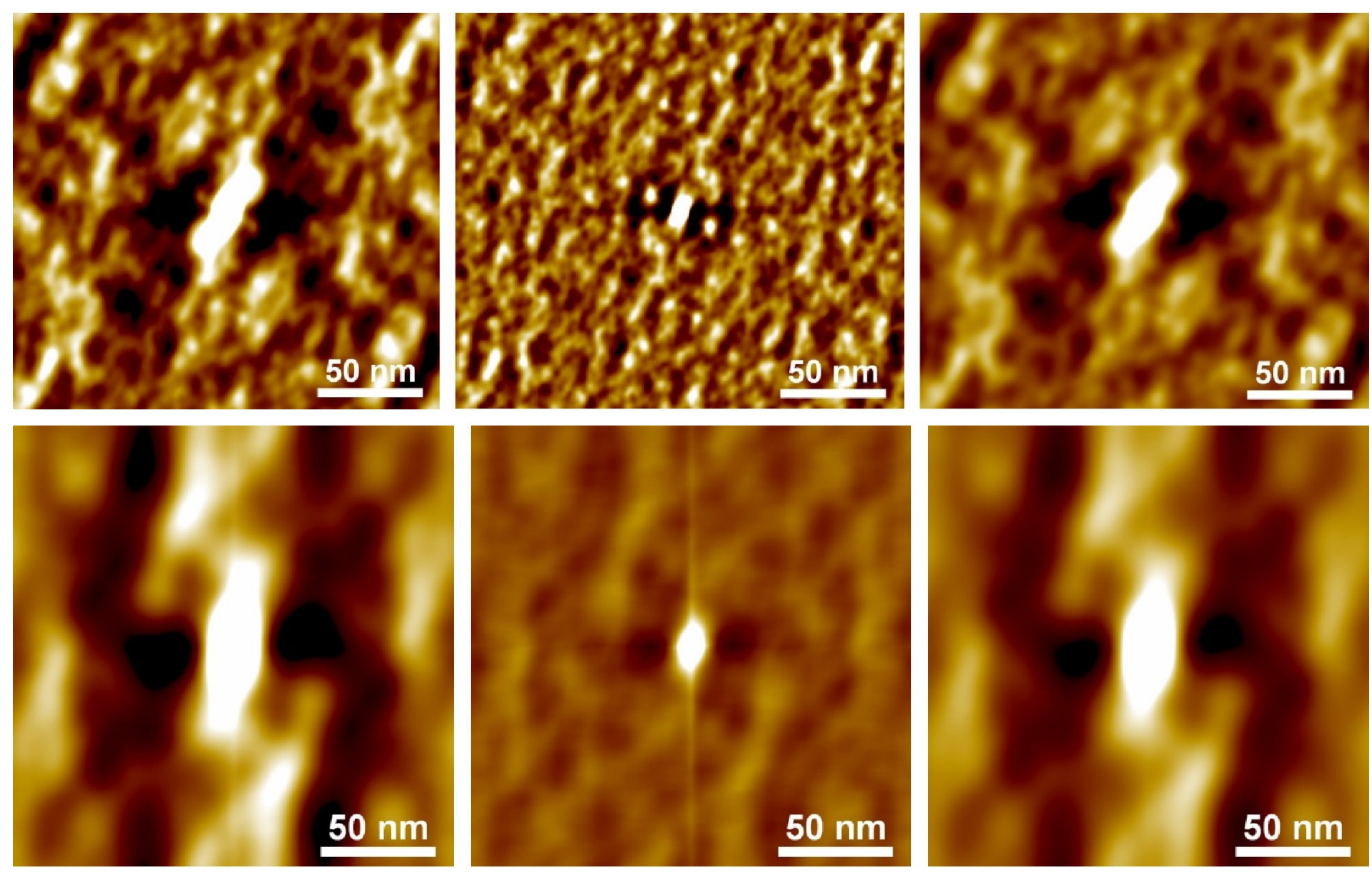

FIG. 7: (Color online) 2D autocorrelation images of graphene (top row) and $\mathrm{SiO}_{2}$ (bottom row). Left: autocorrelation calculated from original images. Middle and right: high-frequency and low-frequency part, obtained by high pass or low pass filtering, respectively, using a $20 \mathrm{~nm}$ first-order Butterworth filter. Note that the color scale is identical within each column. Drift effects have been removed from the graphene images by using the atomic resolution.

within the literature using only the data taken with a similar laser wave length $(514-532 \mathrm{~nm})$ [19, 33, 34, we find $2680-2700 \mathrm{~cm}^{-1}$. Hence, we believe that the major- ity of samples investigated so far exhibit intrinsic rippling as displayed in the right inset of Figure 7a. 

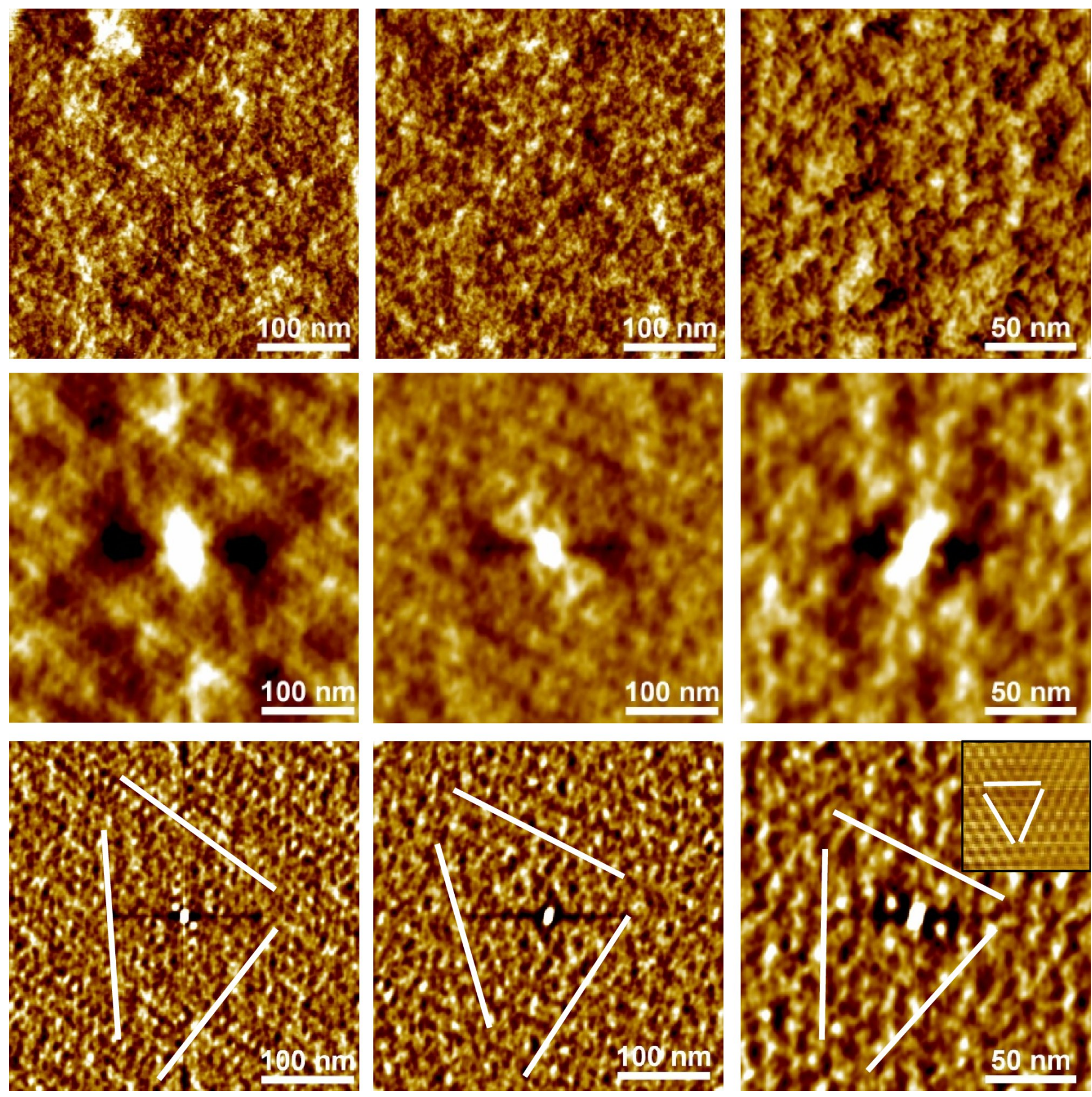

FIG. 8: (Color online) Different graphene areas (top row), separated by several $\mu \mathrm{m}$, with autocorrelation images (middle) and high-pass filtered autocorrelation images (bottom row). The preferential orientation of long-range corrugation varies arbitrarily, while the short-range corrugation shows similar orientation. Inset: Autocorrelation of the atomic resolution image.

[1] N. D. Mermin, Phys. Rev. 176, 250 (1968).

[2] K. S. Novoselov, A. K. Geim, S. V. Morozov, D. Jiang, Y. Zhang, S. V. Dubonos, I. V. Grigorieva, and A. A. Firsov, Science 306, 666 (2004).

[3] Y. Zhang, J. W. Tan, H. L. Stormer, and P. Kim, Nature 438, 201 (2005).
[4] A. K. Geim and K. S. Novoselov, Nature Mat. 6, 183 (2007).

[5] M. I. Katsnelson, K. S. Novoselov, and A. K. Geim, Nature Phys. 2, 620 (2006).

[6] K. S. Novoselov, Z. Jiang, Y. Zhang, S. V. Morozov, H. L. Stormer, U. Zeitler, J. C. Maan, G. S. Boebinger, P. Kim, 

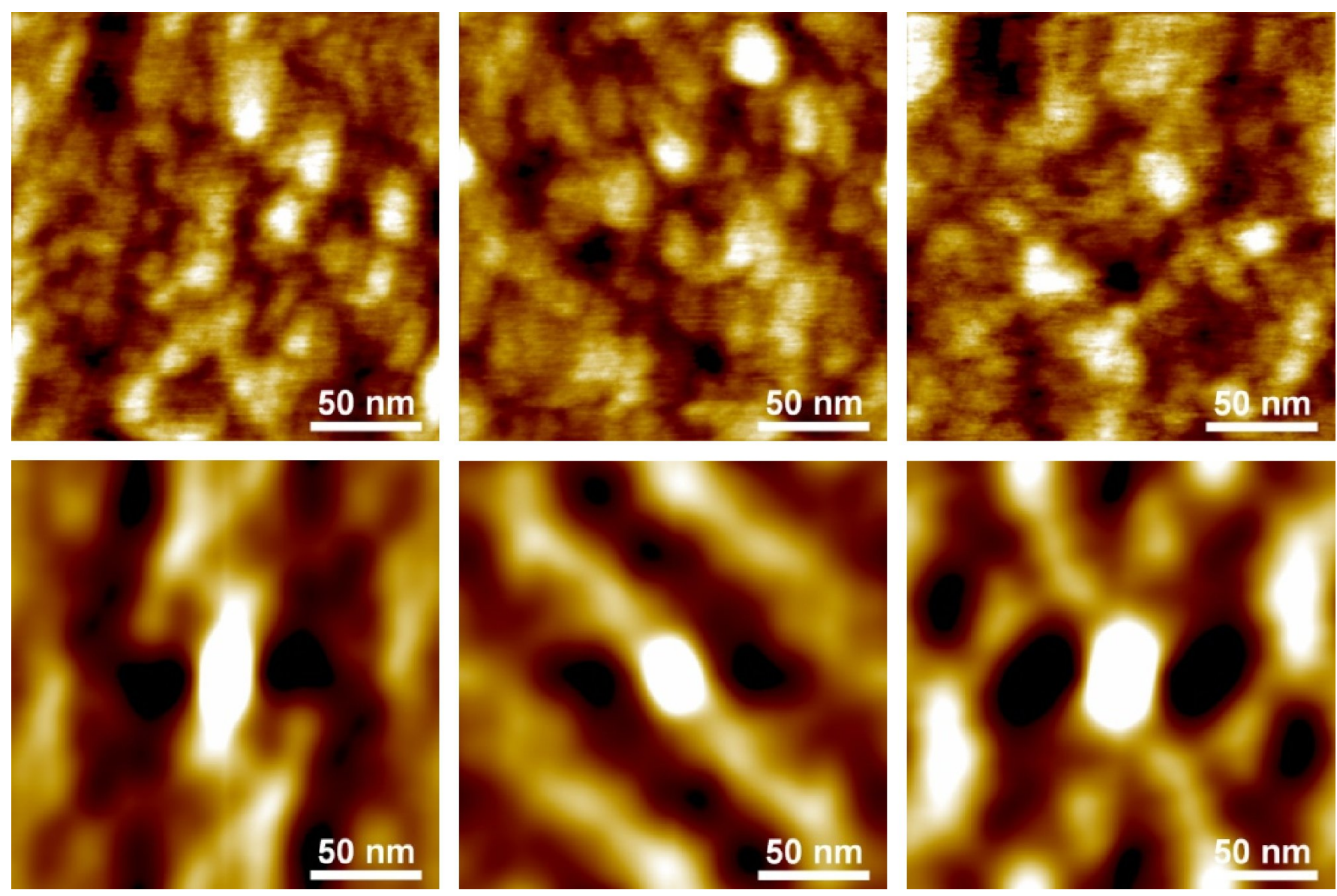

FIG. 9: (Color online) AFM images of different $\mathrm{SiO}_{2}$ areas (top) with corresponding 2D autocorrelation (bottom). The preferential orientation varies arbitrarily.

and A. K. Geim, Science 315, 1379 (2007).

[7] N. Tombros, C. Jozsa, M. Popinciuc, H. T. Jonkman, and B. J. van Wees, Nature 448, 571 (2007).

[8] Y. W. Son, M. L. Cohen, and S. G. Louie, Nature 444, 347 (2006).

[9] S. V. Morozov, K. S. Novoselov, M. I. Katsnelson, F. Schedin, L. A. Ponomarenko, D. Jiang, and A. K. Geim, Phys. Rev. Lett. 97, 016801 (2006).

[10] E. McCann, K. Kechedzhi, V. I. Fal'ko, H. Suzuura, T. Ando, and B. L. Altshuler, Phys. Rev. Lett. 97, 146805 (2006).

[11] J. C. Meyer, A. K. Geim, M. I. Katsnelson, K. S. Novoselov, T. J. Booth, and S. Roth, Nature 446, 60 (2007).

[12] A. Fasolino, J. H. Los, and M. I. Katsnelson, Nature Mat. 6, 858 (2007).

[13] S. V. Morozov, K. S. Novoselov, M. I. Katsnelson, F. Schedin, D. Elias, J. A. Jaszczak, and A. K. Geim, Phys. Rev. Lett. 100, 016602 (2008).

[14] E. Stolyarova, K. T. Rim, S. Ryu, J. Maultzsch, P. Kim, L. E. Brus, T. F. Heinz, M. S. Hybertsen, and G. W. Flynn, Proc. Natl. Acad. Sci. 104, 9209 (2007).

[15] M. Ishigami, J. H. Chen, W. G. Cullen, M. S. Fuhrer, and E. D. Williams, Nano Lett. 7, 1643 (2007).

[16] F. V. Tikhonenko, D. W. Horsell, R. V. Gorbachev, and A. K. Savchenko, Phys. Rev. Lett. 100, 056802 (2008).
[17] Y. Zhang, V. W. Brar, F. Wang, C. Girit, Y. Yayon, M. Panlasigui, A. Zettl, and M. F. Crommie, arXiv:0802.4315v1 (2008).

[18] M. C. Lemme, T. J. Echtermeyer, M. Baus, and H. Kurz, IEEE Elect. Dev. Lett. 28, 282 (2007).

[19] A. C. Ferrari, J. C. Meyer, V. Scardaci, C. Casiraghi, M. Lazzeri, F. Mauri, S. Piscanec, D. Jiang, K. S. Novoselov, S. Roth, et al., Phys. Rev. Lett. 97, 187401 (2006).

[20] M. Lemme, T. Echtermeyer, M. Baus, B. Szafranek, J. Bolten, M. Schmidt, T. Wahlbrink, and H. Kurz, SolidState Electronics 52, 514 (2008).

[21] Y. W. Tan, Y. Zhang, K. Bolotin, Y. Zhao, S. Adam, E. H. Hwang, S. Das Sarma, H. L. Stormer, and P. Kim, Phys. Rev. Lett. 99, 246803 (2007).

[22] J. Wiebe, A. Wachowiak, F. Meier, D. Haude, T. Foster, M. Morgenstern, and R. Wiesendanger, Rev. Sci. Instrum. 75, 4871 (2004).

[23] V. Geringer, S. Runte, M. Liebmann, and M. Morgenstern, to be published.

[24] MikroMasch (www.spmtips.com), NSC15 (Si) and DP15/HiRes-W/AlBS (Si cantilever with W tip apex).

[25] H. Hölscher and U. D. Schwarz, Int. J. Non-Linear Mech. 42, 608 (2007).

[26] C. Wittneven, R. Dombrowski, M. Morgenstern, and R. Wiesendanger, Phys. Rev. Lett. 81, 5616 (1998). 


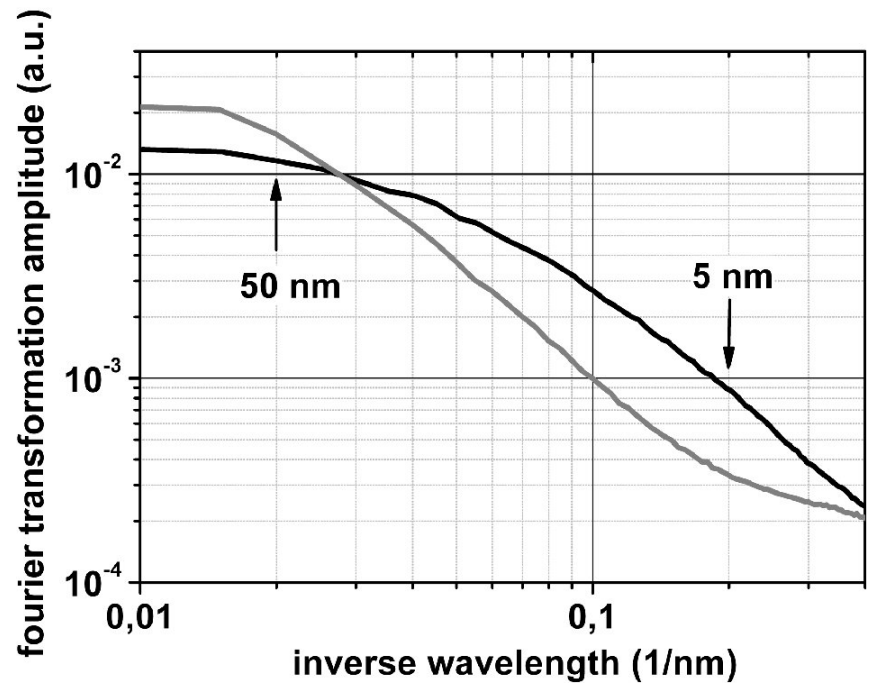

FIG. 10: Radial averages of the Fourier transformations of graphene (black) and $\mathrm{SiO}_{2}$ (grey) images, averaged over 10 images. The crossing point of individual curves varies between 20 and $40 \mathrm{~nm}$.
[27] J. A. Stroscio, R. M. Feenstra, and A. P. Fein, Phys. Rev. Lett. 57, 2579 (1986).

[28] V. W. Brar, Y. Zhang, Y. Yayon, T. Ohta, J. L. McChesney, A. Bostwick, E. Rotenberg, K. Horn, and M. F. Crommie, Appl. Phys. Lett. 91, 122102 (2007).

[29] G. M. Rutter, J. N. Crain, N. P. Guisinger, T. Li, P. N. First, and J. A. Stroscio, Science 317, 219 (2007).

[30] P. Lauffer, K. V. Emtsev, R. Graupner, T. Seyller, L. Ley, S. A. Reshanov, and H. B. Weber, Phys. Rev. B 77, 155426 (2008).

[31] I. Horcas, R. Fernández, J. M. Gómez-Rodríguez, J. Colchero, J. Gómez-Herrero, and A. M. Baro, Rev. Sci. Instrum. 78, 013705 (2007).

[32] See supplementary information.

[33] A. Gupta, G. Chen, P. Joshi, S. Tadigadapa, and P. Eklund, Nano Letters 6, 2667 (2006).

[34] D. Graf, F. Molitor, K. Ensslin, C. Stampfer, A. Jungen, C. Hierold, and L. Wirtz, Nano Letters 7, 238 (2007). 


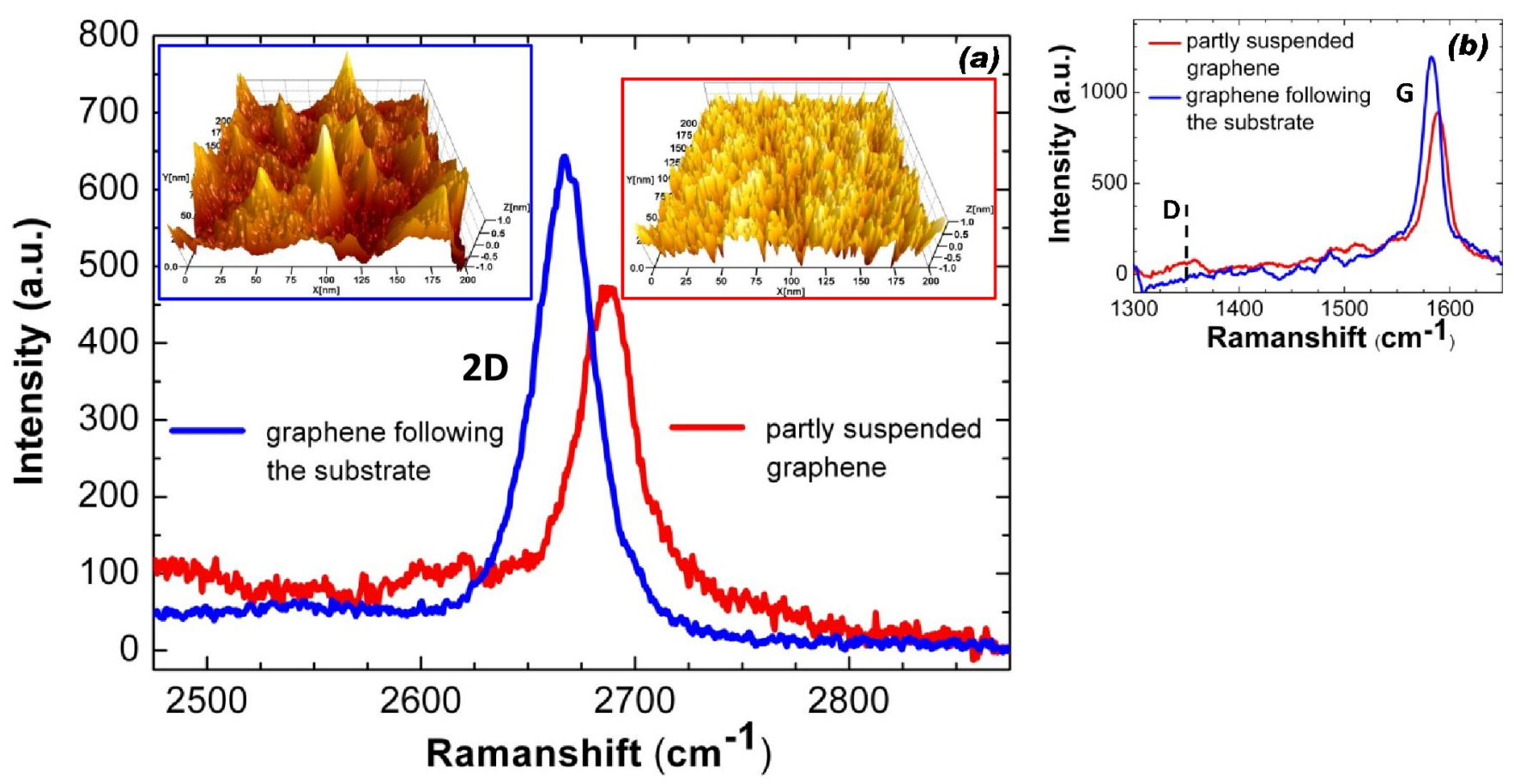

FIG. 11: (Color online) (a) Comparison of Raman spectra at $532.1 \mathrm{~nm}$ laser wave length for partly suspended and following the substrate graphene monolayer flake. The $2 \mathrm{D}$ peak position is $2686.7 \mathrm{~cm}^{-1}$ for partly suspended graphene and $2670.5 \mathrm{~cm}^{-1}$ for the flake following the substrate. The experimental conditions for both samples are identical. Left inset: STM measurement of the graphene flake following the substrate $(0.4 \mathrm{~V}, 0.2 \mathrm{nA})$. Right inset: STM measurement of the partly suspended graphene flake $(1 \mathrm{~V}, 0.2 \mathrm{nA})$. (b) Raman spectra of the D and G band for the same samples. The D line intensity $\left(1350 \mathrm{~cm}^{-1}\right.$, marked by a dashed line) is very weak for both samples. The $G$ line from the flake following the substrate is shifted to lower values by $5.5 \mathrm{~cm}^{-1}$ with respect to the $\mathrm{G}$ peak of the partly suspended graphene. 